\title{
Polyimide-Based Capacitive Humidity Sensor
}

\author{
Jamila Boudaden ${ }^{1,2, *} \mathbb{1}$, Matthias Steinmaß1 1,3,4 , Hanns-Erik Endres ${ }^{1}$, Andreas Drost ${ }^{1}$, \\ Ignaz Eisele ${ }^{1}$, Christoph Kutter ${ }^{1,4}$ and Peter Müller-Buschbaum ${ }^{3}$ \\ 1 Fraunhofer EMFT, Research Institution for Microsystems and Solid State Technologies EMFT, \\ Hansastraße 27d, D-80686 Munich, Germany; matthias.steinmassl@emft.fraunhofer.de (M.S.); \\ hanns-erik.endres@emft.fraunhofer.de (H.-E.E.); andreas.drost@emft.fraunhofer.de (A.D.); \\ ignaz.eisele@emft.fraunhofer.de (I.E.); christoph.kutter@emft.fraunhofer.de (C.K.) \\ 2 Institute of Electronic and Sensor Materials, Technische Universität Bergakademie Freiberg, \\ Gustav-Zeuner-Str. 3, D-09599 Freiberg, Germany \\ 3 Physik-Department, Lehrstuhl für Funktionelle Materialien, Technische Universität München, \\ James-Franck-Strasse 1, D-85748 Garching, Germany; muellerb@ph.tum.de \\ 4 Physik-Department, Universität der Bundeswehr München, Werner-Heisenberg-Weg 39, \\ D-85579 Neubiberg, Germany \\ * Correspondence: jamila.boudaden@emft.fraunhofer.de; Tel.: +49-89-54759-161
}

Received: 4 March 2018; Accepted: 3 May 2018; Published: 11 May 2018

check for updates

\begin{abstract}
The development of humidity sensors with simple transduction principles attracts considerable interest by both scientific researchers and industrial companies. Capacitive humidity sensors, based on polyimide sensing material with different thickness and surface morphologies, are prepared. The surface morphology of the sensing layer is varied from flat to rough and then to nanostructure called nanograss by using an oxygen plasma etch process. The relative humidity (RH) sensor selectively responds to the presence of water vapor by a capacitance change. The interaction between polyimide and water molecules is studied by FTIR spectroscopy. The complete characterization of the prepared capacitive humidity sensor performance is realized using a gas mixing setup and an evaluation kit. A linear correlation is found between the measured capacitance and the RH level in the range of 5 to $85 \%$. The morphology of the humidity sensing layer is revealed as an important parameter influencing the sensor performance. It is proved that a nanograss-like structure is the most effective for detecting $\mathrm{RH}$, due to its rapid response and recovery times, which are comparable to or even better than the ones of commercial polymer-based sensors. This work demonstrates the readiness of the developed RH sensor technology for industrialization.
\end{abstract}

Keywords: humidity sensor; flat polyimide; nanograss polyimide; rough polyimide

\section{Introduction}

Humidity is defined as the water vapor amount in air. It is the most abundant greenhouse gas and an important abiotic factor influencing the human life. To describe humidity in a precise way, several items are commonly used, which are absolute humidity $(\mathrm{AH})$, relative humidity $(\mathrm{RH})$, specific humidity (SH), and dew point [1]. The RH term is commonly employed to describe the content of water vapor in the surrounding atmosphere by taking into consideration both temperature and pressure.

Recently, the state of the art of humidity monitoring has evolved rapidly and continuously. However, the wide application range of devices being able to record humidity is behind the intensive scientific studies dedicated to this area. Their most important fields of use are indoor application, plants growth in agricultural market, and control of industrial processes, electronics, and semiconductor area, as well as medical processes [2-8]. 
A survey of the literature leads to a classification of humidity sensors based on their working principle as capacitive, electrical conductivity (or resistive) and optical sensors. It is obvious that, except optical sensors, a humidity sensor is defined as a combination of a transducer and a sensing material to humidity.

Interdigitated transducers (IDT), also known as interdigital electrodes, have been intensively studied and used for unlimited applications due to the possibility of measuring both capacitance and resistance $[9,10]$. An IDT is a structure consisting of two comb-like electrodes on a planar substrate. Their technological realization is readily mastered in the semiconductor field. To design a relative humidity sensor by considering the IDTs as transducer, three main sensing materials were often reported by the scientific community: ceramic, semiconductor, and polymer materials [7].

Ceramic materials, like $\mathrm{Al}_{2} \mathrm{O}_{3}, \mathrm{TiO}_{2}, \mathrm{SiO}_{2}$, and spinel compounds with $\mathrm{AB}_{2} \mathrm{O}_{4}$ structural composition, are known to change their electrical properties, such as resistance, in the presence of humidity [7]. The first initial adsorbed layer of water molecules is stable and ordered. A chemical reaction between water molecules and the metal oxide surface was suggested to form two hydroxyls group [11]. Each single water molecule is attached to two neighboring hydroxyls by hydrogen bonding [12], thus a second water layer is formed. By further adsorption of water vapor, additional layers of water molecules bond by weak forces (van der Waals-type) in a disordered manner on the surface of the first physically adsorbed layer. Hence, proton conduction takes place from one oxygen atom (in a water molecule) to the next one, through the Grotthus mechanism [13]. This mechanism requires disorder, which is not obtained during the formation of the first chemisorbed and the first physisorbed layers. Therefore, the conductivity mechanism starts upon the second physisorbed layer. Consequently, at low humidity, the conductivity is almost unchanged. For this reason, most ceramic materials are not sensitive to RH below 20\% [14]. Decreasing the ceramic's pore size enhances the sensitivity at lower humidity level. Unfortunately, this decrease goes ahead with a faster degradation due to clogged small pores with dirt.

Semiconducting materials, like $\mathrm{SnO}_{2}, \mathrm{In}_{2} \mathrm{O}_{3}$ [15], and perovskites [16], are promising candidates for sensing humidity with a high precision. Existing research activities focus mostly on n-type semiconductors, for which the conductivity increases with increasing humidity. Semiconductors show physical adsorption of water, where two mechanisms contribute to the change in its electrical conductivity. The adsorption happens at dangling bonds of the semiconductor surface molecules or by van der Waals interaction to surface molecules. Considering a physisorbed water molecule at room temperature, the electronegativity of oxygen is higher than that of hydrogen. Thus, there are positive partial charges at the hydrogen atoms. The positive side of the physisorbed water dipoles attracts electrons to the surface of the semiconductor [7]. This leads to a bending down of the conduction and valence band at the surface. At higher temperatures, oxygen molecules react with the surface molecules and are reduced to oxygen ions that are chemisorbed to the semiconductor surface. Thus, electrons are accumulated at the surface, which results in an increase of the number of holes, leading to band bending at semiconductor surfaces. Water molecules, in the nearby area, are physisorbed to the semiconductor surface and replace the oxygen ion sites. The surface electrons depletion is neutralized. Hence, conductivity is increased. Semiconductor based humidity sensors are usually driven at temperatures around $200{ }^{\circ} \mathrm{C}$ because the signal change is higher and linear [17].

The third type of humidity-sensing material is an organic material being commonly a polymer. It is important to underline that polymers are an emerging candidate in many sensing applications due to low cost, commercial availability, and easy deposition on different transducers. Besides this, a photosensitive polymer adds advantages related to rapid polymerization, elimination of volatile organic solvents usage, and reduction of technological process steps. A polymeric layer in contact with water vapor shows a change in its conductivity or its dielectric constant $\varepsilon$. As water diffuses into a hydrophobic polymer layer like polyimide, poly(methyl methacrylate) (PMMA), cellulose acetate butyrate (CAB), or poly(ethyleneterephthalate) (PETT), the matrix absorbs water and only its relative permittivity $\varepsilon$ undergoes changes [7]. Several studies demonstrate that hygroscopic polymer sensing 
layers present a good sensitivity to humidity at room temperature [14,18]. A linear sensing performance between $5 \%$ and $90 \% \mathrm{RH}$ and a long-term stability has been shown due to the establishment of cross-linking and interpenetrating networks (IPN) [19].

Nowadays, capacitive humidity sensors dominate the market. Polymeric layers as a sensitive layer in combination with interdigitated electrodes are intensively studied [7]. Despite the availability of several low-cost relative humidity sensors on the market, new small and medium size companies are looking for mature technology for realizing RH sensors for a wide range of applications. Although polyimide films have been used as sensing layers for humidity sensors [18,20-27], complete reports about the properties of polyimide based capacitive for use as the sensing layer for $\mathrm{RH}$ are still missing. In the present work, we report detailed insights into the performance of a planar interdigitated transducer coated with polyimide sensing material having different thickness and morphologies. It is worth mentioning that planar IDTs take advantage only of the electric field lines penetrating the polymeric layer (in general the upper half of the transducer).

\section{Experimental Details}

A chemical sensor is a device that transforms chemical information, i.e., the analyte concentration, into an electrically useful signal. Basically, it consists of a transducer and a sensitive layer. The chemical information is obtained either due to a chemical reaction or due to a sorption process between the analyte and the sensitive layer. In our study, we fabricated capacitive humidity sensors, which monitor any changes of in the dielectric properties of the appropriate sensing material. The transducer was a pair of planar interdigitated electrodes. The sensing material was polymer-based material called polyimide.

\subsection{Transducer Configuration}

A pair of interdigitated microelectrodes was produced by standard photolithography on a $620 \mu \mathrm{m}$ thick glass substrate. For operating at elevated temperatures, quartz substrates are the best choice. After cleaning the glass substrate wafer, a thin adhesion layer of TiW $(40 \mathrm{~nm})$ followed by a thick gold layer $(140 \mathrm{~nm})$ were deposited by sputtering deposition technique. The electrodes were patterned by photolithography technique using the desired mask. The pattern included two wires around the interdigitated electrodes, one to heat the sensor and the other to measure the surrounding temperature (Figure 1). Each chip featured two interdigitated gold electrodes with filling area of $2 \times 2 \mathrm{~mm}^{2}$. Finger width, length, and pitch were set to $6 \mu \mathrm{m}, 2 \mathrm{~mm}$, and $12 \mu \mathrm{m}$, resulting in 83 and 42 fingers, respectively.

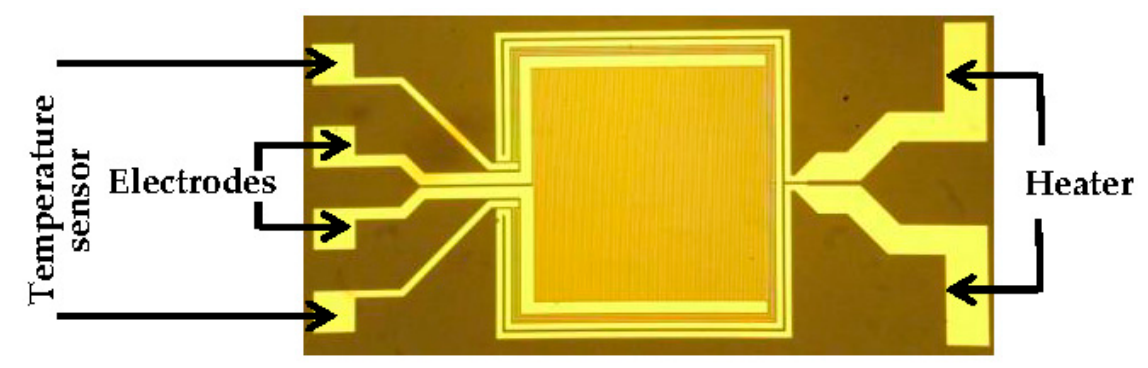

Figure 1. Layout of studied interdigitated transducer. The electrodes are surrounded by a temperature sensor and a heater.

\subsection{Sensing Material Preparation}

The polyimide used for humidity sensing layers was prepared from a photosensitive solvent developable polyamide acid ester. First, the substrate surface was cleaned with a stripper in an ultrasonic bath from any contaminants and organic impurities prior to the coating process in order to ensure a good adhesion of the sensing layer to the glass and metallic electrodes surface. The polyimide 
material was spin coated homogeneously on top of the planar electrodes on the 6-inch glass wafer. Different layer thicknesses were obtained, ranging from $1 \mu \mathrm{m}$ to $11 \mu \mathrm{m}$. The spin coating parameters (spin speed and time) varied according to the desired final film thickness and the precursor initial viscosity. After spin coating, the films underwent a soft bake for a short period of time to drive out residual solvent. Then, the photosensitive layer was exposed to an appropriate dose using a mask for pad opening. The exposure dose was chosen based on the thickest layer in case of a variation of the coating thickness on the substrate. The wet development process was realized at room temperature for an optimal time and followed by flood exposure, without a mask, applied after developing to allow a complete crosslinking reaction. The imidization reaction took place between the amino group and the ester group resulting in an imine group. After completing the photolithographic process, the film was cured in a Yes Oven under $\mathrm{N}_{2}$ inert atmosphere. The curing temperature was $400^{\circ} \mathrm{C}$.

\subsection{Transducer and Sensing Material}

The coated wafer with polyimide sensing material was diced into $4.7 \times 3.9 \mathrm{~mm}^{2}$ large individual chips. Each chip was mounted on an adapted printed circuit board (PCB), glued, and then gold wire bonded thereto. The PCB was equipped with zero insertion force (ZIF) connectors.

\subsection{Gas Mixing Apparatus}

A reliable gas mixing apparatus, which is a part of a gas measurement setup, is an important system for characterizing gas sensors under desired gas mixtures. The dynamic volumetric method, described in reference [28], was adopted to generate a defined gas mixture in a reproducible way. For this study, the gas mixing apparatus was able to generate a constant level of absolute humidity by selecting the adopted ratio between the volumes of humid gas to the carrier gas, which is $\mathrm{N}_{2}$ or synthetic air.

\subsection{Capacitance Measurements Using an Impedance Analyzer}

Up to 10 chips could be placed into a cylindrical stainless steel chamber (sensor chamber), which is connected to the gas mixing apparatus (Figure 2a). Consequently, they were simultaneously characterized under the same gas environment conditions. A radial distribution of the gas and isolating wall between the 10 chips ensured a continuous and controlled flow of the gas.

The electrical properties of the sensors were monitored by impedance spectroscopy using a Solartron gain-phase frequency analyzer model 1260 A controlled by a PC, permitting automated data collection. The impedance measurements could be carried out at different temperatures (from RT to $200{ }^{\circ} \mathrm{C}$ ) by heating up the chip using the integrated heater around the electrodes. The measurements were performed from $1 \mathrm{MHz}$ to $10 \mathrm{~Hz}$ to get the optimal frequency at which the sensor capacitance change linearly as a function of $\mathrm{RH}$. Within another project, it is planned to develop a miniaturized system using the AD chip. The AC Voltage excitation of the AD chip is around $38 \mathrm{kHz}$. For this reason, most of the experimental measurements are done at $40 \mathrm{kHz}$.

For this study, the capacitance is recorded at $25^{\circ} \mathrm{C}$ under different frequencies [29]. The sensor is subjected to a dynamic change of relative humidity levels and its capacitance is monitored. Most of the developed sensors capacitance were monitored under relative humidity levels ranging from $5 \%$ to $85 \%$ at $25{ }^{\circ} \mathrm{C}$. The lower measured capacitance between $5 \%$ and $15 \%$ is considered as a base capacitance. The error bars of the capacitance are related to the impedance analyzer error.

\subsection{Capacitance Measurements Using Evaluation Kit}

An evaluation kit with high speed data acquisition was designed. Additional features are low power, low cost, compact size, and network compatibility. The used microcontroller STM32-L476RG possesses a low-power design, several Analog-to-Digital converters (ADC), and a touch sensing controller for capacitive measurements via the charge transfer method on board. The touch sensing controller consists of a number of internal switches. After loading the sensor capacitor with a constant 
voltage, the charge is transferred by the switches to an external reference capacitor (104 times bigger). This process is repeated $\mathrm{N}$ times, until a threshold voltage is reached on the reference capacitor. The capacitance of the sensor is then calculated by [30]

$$
C_{s}=-\frac{C_{R E F}}{N} \ln \left(1-\frac{V_{t h}}{V_{d d}}\right)
$$

where:

$V_{t h}:$ threshold voltage $(1.5 \mathrm{~V})$

$V_{d d}$ : positive supply voltage $(3.3 \mathrm{~V})$

$C_{R E F}:$ reference capacitor

$N$ : number of charge transfer cycles needed to charge the $C_{s}$.

An extension circuit board was developed, which holds sensor connectors, heater driver circuit, resistance temperature detectors (RTD), and reference capacitors for the charge transfer method. Additionally, PT1000 and SHT25 were added as reference sensors and connectors for communication protocols (UART and I2C). The evaluation kit can simultaneously measure three sensors by measuring the capacitance and controlling the operating temperature by a proportional-integral-derivative (PID) algorithm. It permitted measuring the response and recovery times of the developed sensors by ensuring a short measurement interval and quick gas exchange ability. In order to use the evaluation kit together with the gas mixing apparatus, a PEEK lid was designed and screwed with a sealing ring onto the PCB, where three sensors sockets were positioned (Figure 2b). The sampling time was $150 \mathrm{~ms}$. Therefore, the evaluation kit permitted measuring the response and recovery times of the developed sensors due to a short measurement interval and quick gas exchange ability.

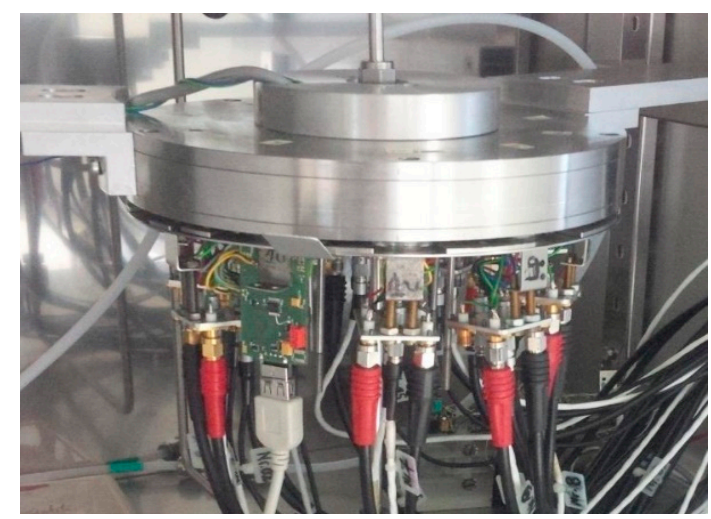

(a)

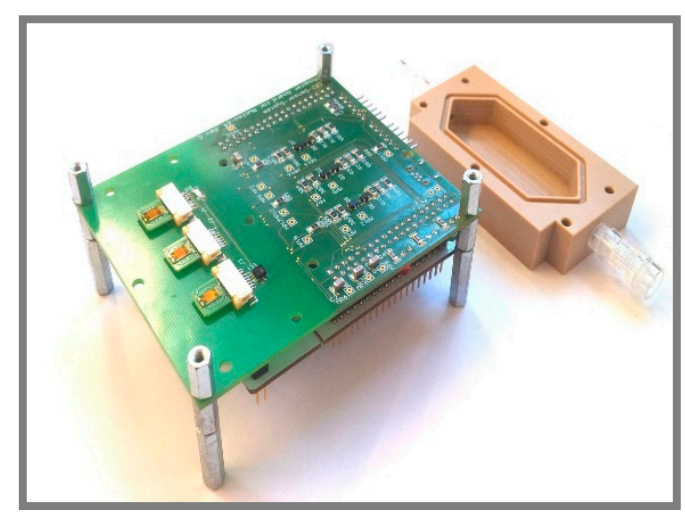

(b)

Figure 2. Photograph of (a) sensor chamber for the impedance analysis and (b) evaluation kit with a PEEK lid. Both can be connected to the gas mixing apparatus.

\section{Results and Discussion}

\subsection{FTIR Characterization}

Fourier-transform infrared spectroscopy (FTIR) is an extremely reliable method that provides chemical bond and molecular composition present of any partially transparent material. To realize FTIR spectroscopy for our purpose, silicon test wafer substrates $\left(1 \times 1 \mathrm{~cm}^{2}\right)$ were coated with a polyimide layer and examined under IR radiation at different humidity levels by a FTIR spectrometer. A background reference curve is measured with an uncoated sample from the same silicon wafer. A first measurement is performed with a polyimide coated sample under dry condition inside the FTIR interior space at nearly $0 \% \mathrm{RH}$ (grey curve in Figure 3). The same sample was then interconnected to a 
copper humidity chamber and placed in the FTIR beam path. The heatable copper chamber has a small water reservoir at the bottom. A thin tube, with a $1 \mathrm{~mm}$ diameter, ensures the connection between the chamber and the water reservoir. The chamber is equipped with two $\mathrm{ZnS}$ windows having strong IR transmitting properties. After closing the chamber, water molecules from the reservoir evaporate and diffuse through the thin tube into the chamber, where the sample coated with polyimide is placed. After $24 \mathrm{~h}$, the relative humidity reached $50 \%$. At this time, a second FTIR measurement is performed (purple curve). Both infrared spectra reveal a wave like interference pattern, which are visible especially in the range of 3000 to $1750 \mathrm{~cm}^{-1}$. Polymer-based sensors are usually based on the analytes diffusion into the polymer sensing matrix. FTIR spectra of a polyimide layer at $0 \%$ and $50 \%$ relative humidity reveal the absence of any chemical reaction between the polyimide layer and the water vapor molecules, which is infrared-sensitive in the range between 8000 and $800 \mathrm{~cm}^{-1}$. Therefore, chemisorption of water molecules with the polyimide matrix can be ruled out. The interaction of polyimide with water molecules is purely a physical absorption. Moreover, the FTIR spectra show the presence of several imide peaks, especially at about $1720 \mathrm{~cm}^{-1}, 1780 \mathrm{~cm}^{-1}$, and at $1350 \mathrm{~cm}^{-1}$, which belong to $\mathrm{C}-\mathrm{N}$ stretching vibration of the imide ring. This confirms that a successful imidization has been reached and finally an amorphous stable material is formed.

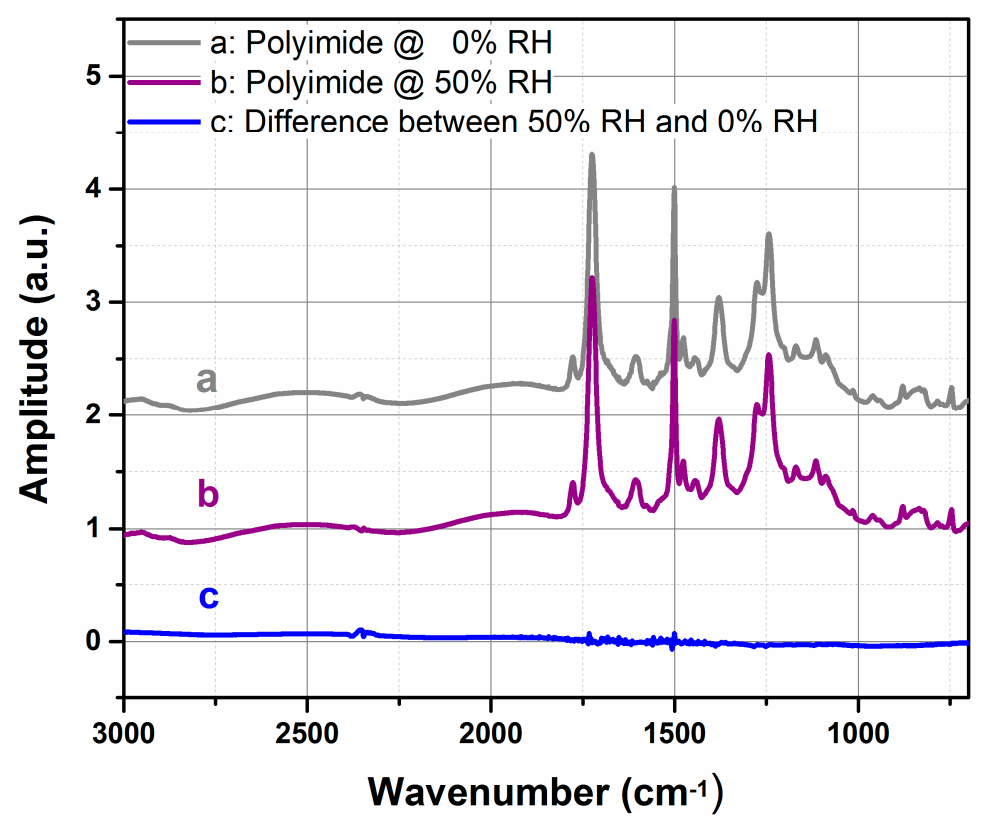

Figure 3. Two infrared spectra covering a range from 3000 and $800 \mathrm{~cm}^{-1}$ measured at zero RH (grey) and $50 \% \mathrm{RH}$ (purple). The difference spectrum of both spectra is shown for clarity (blue). Between 3000 and $1800 \mathrm{~cm}^{-1}$, reflections on the flat polyimide surface cause interference. The small peaks at $2300 \mathrm{~cm}^{-1}$ result from remaining gaseous $\mathrm{CO}_{2}$ inside the FTIR chamber. $\mathrm{RH}$, relative humidity.

\subsection{Effect of Frequency Using Impedance Analyzer}

The electrical properties of interdigitated transducer coated with polyimide, under different relative humidity levels, are monitored by impedance spectroscopy in the frequency range of $10 \mathrm{~Hz}$ to $1 \mathrm{MHz}$. The sensor chamber is situated inside a constant temperature incubator at $25^{\circ} \mathrm{C}$. Figure 4 represents the capacitance deduced from the complex impedance using resistor-capacitor (RC) parallel equivalent circuit versus the frequency for different relative humidity. Between $1 \mathrm{kHz}$ and $1 \mathrm{MHz}$, polarization effects of water molecules do not contribute to the sensor's capacitance and the measurement error is minimal. Thus, a linear behavior of the capacitance is obtained (Figure 4). The same constant trend of capacitance was also measured between $100 \mathrm{kHz}$ and $10 \mathrm{MHz}$ [24], where metallic interdigitated electrodes were printed on one side of a polyimide substrate. For extensive 
studies, $40 \mathrm{kHz}$ was chosen as the measurement frequency for the impedance analysis. At $40 \mathrm{kHz}$, the error of the impedance analyzer is small. Additionally, the polarization of water molecules does not contribute to the impedance, as it is the case at high humidity levels and small frequencies.

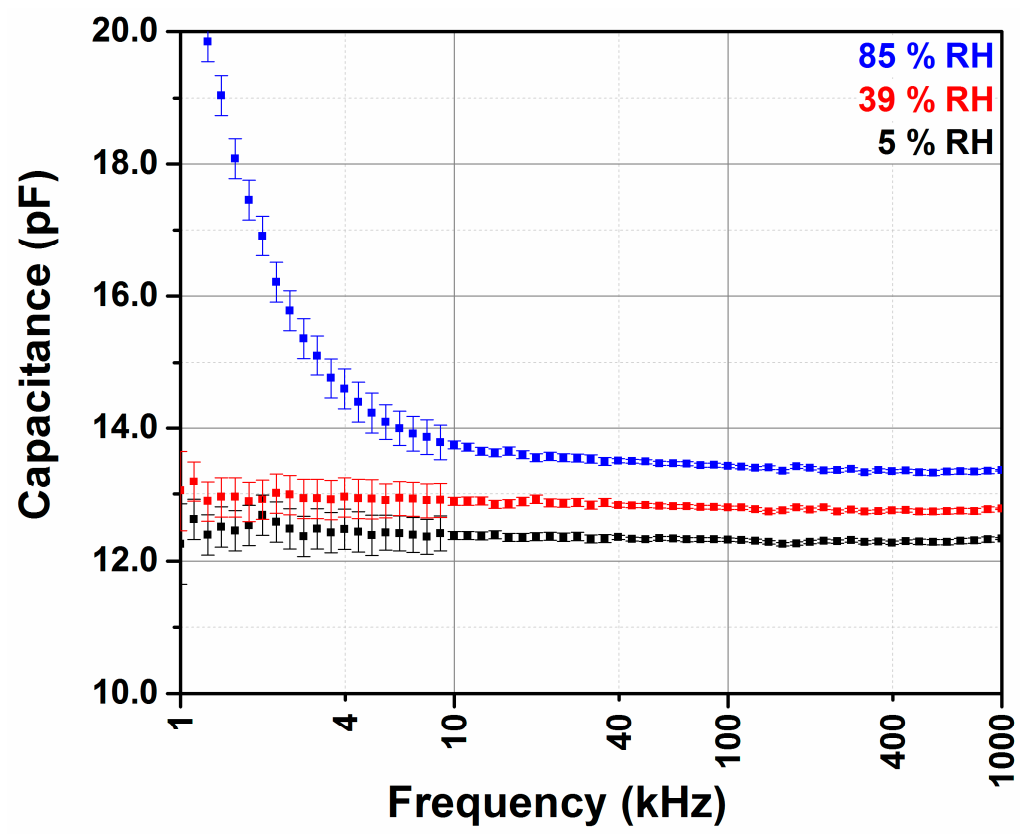

Figure 4. Frequency-dependent capacitance for different $\mathrm{RH}=5 \%$ (black), 39\% (red), and 85\% (blue) of a $11 \mu \mathrm{m}$ thick polyimide layer. The Error bars represent the uncertainty given by the impedance analyser.

\subsection{Dynamic Mode Using the Impedance Analyzer}

The 4.6 and $11 \mu \mathrm{m}$ thick polyimide films are deposited onto the IDTs. The capacitance is monitored as a function of time by an impedance analyzer at $40 \mathrm{kHz}$ frequency under different $\mathrm{RH}$ levels. Figure 5a displays the typical response and recovery curves of the as-fabricated humidity sensor to different amount of relative humidity in the carrier gas. The sensors are alternatively exposed to different $\mathrm{RH}$, which are varied from $16 \%$ to $85 \%$. The exposure time of the sensor to the RH, higher than background value $16 \%$, is fixed to $20 \mathrm{~min}$. Once the RH is decreased to the background value $16 \%$, the sensors capacitance returns to the initial base capacitance in a reversible without observing any pronounced hysteresis.
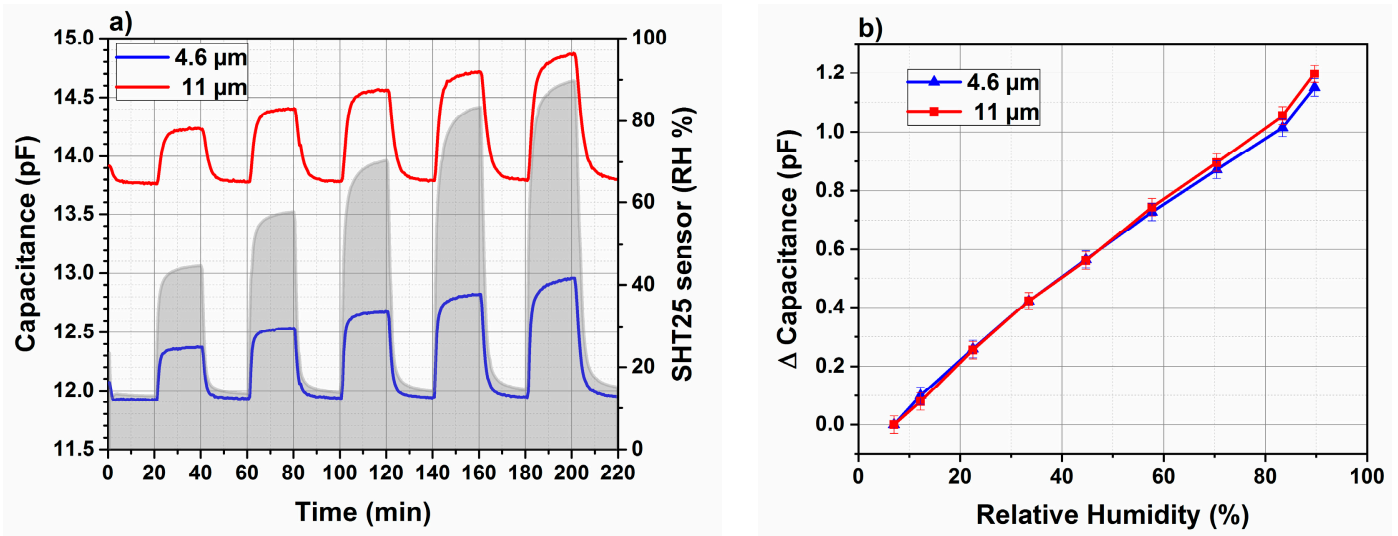

Figure 5. (a) Temporal evolution of capacitance of $4.6 \mu \mathrm{m}$ and $11 \mu \mathrm{m}$ polyimide layers at different $\mathrm{RH}$ measured by SHT25 commercial sensor. (b) Calibration curve of 4.6 (blue) and $11 \mu \mathrm{m}$ (red) polyimide layers. The capacitance change is traced versus relative humidity at a frequency of $40 \mathrm{kHz}$. 
Figure $5 \mathrm{~b}$ shows the capacitance reached after exposing the sensors to different $\mathrm{RH}$ for $20 \mathrm{~min}$. The capacitance versus different $\mathrm{RH}$ values increases monotonically by increasing the RH from $10 \%$ to $80 \%$ for polyimide layers with two different thicknesses. The RH is also determined using commercial SHT25 sensor. Both developed humidity sensors represent a good linearity with respect to variations in relative humidity. It is also observed that the capacitance changes of IDTs coated with two different polyimide thicknesses $4.6 \mu \mathrm{m}$ and $11 \mu \mathrm{m}$ are identical. As the capacitance change results from a bulk absorption of water molecules a decrease of the capacitance change would have been expected as the polyimide thickness decreases. Therefore, a complementary study is necessary to investigate the penetration depth of the electrical field lines by the mean of FEM software to explain the obtained calibration curves for different polyimide thicknesses.

Above $80 \%$ and below $15 \%$, a deviation from the linear behavior should be expected. In addition, the hysteresis characteristic of the polyimide-based humidity sensor was obtained in static mode. The RH was increased continuously from $12 \%$ to $85 \%$ by a step of $10 \%$. Then, $\mathrm{RH}$ has to be decreased from $85 \%$ to $12 \%$ by a step of $10 \%$. SHT 25 gave the reference measurement of the humidity. The hysteresis is evaluated to be less than $2 \%$.

\subsection{Penetration Depth Using FEM Simulation}

COMSOL Multiphysics (version 4.3a) is a simulation software, which applies finite element simulation to a 3D model of the structure to be studied in this work. The CAD is used to build a 3D model that consists of several layers: glass substrate, gold layer, sensitive layer (optional), and air layer. The derived capacitance of a bare IDT by FEM simulations agree well with the one measured by the impedance analyzer. By combining simulations with different dielectric constants of the sensitive layer and the measured sensitivity of the coated IDT, the correlation between the dielectric constant of the polyimide layer and the relative humidity was determined. Therefore, at $25 \% \mathrm{RH}$ the dielectric constant of polyimide is equal to 3.5 .

Calculation of the penetration depth is simulated to determine the maximum reasonable polyimide layer thickness. Figure 6a shows stream lines of the electric field for used IDTs having a space width between consecutive electrodes of $6 \mu \mathrm{m}$ and an electrode width of $6 \mu \mathrm{m}$. The simulated structure is identical to the one used as a transducer for the developed humidity sensor. Stream lines of the electric field leave the electrode perpendicular to the surface. The electric field lines form ellipsoids going from positive to negative electrodes. As a consequence, IDTs are coated with a polyimide sensitive layer. The 3D models for the FEM simulations consist of a $4.6 \mu \mathrm{m}$ thick polyimide layer on the top of IDTs. The dielectric constant of polyimide is higher than that of air $\left(\varepsilon_{\mathrm{PI}} \sim 3.5\right)$. The values of the penetration depth are obtained from COMSOL plots of the electric field. Figure $6 \mathrm{~b}$ shows a plot of the electric field strength along a perpendicular axis to the IDT's planar surface and situated in the middle between two adjacent electrodes. The maximum of the electric field strength is reached at $\mathrm{z}=-0.1 \mu \mathrm{m}$, which is located in the middle of the gold electrodes. Negative $z$ values represent the positions inside the glass substrate and positive $\mathrm{z}$ values those inside the sensing layer. The electric field tends to zero if the $\mathrm{z}$ values tend to infinity. The penetration depth is defined as the depth at which the electric field strength falls below E/e 0.37 E (grey area). The graph, in Figure 5b, represents an IDT with $6 \mu \mathrm{m}$ electrode space and $4.6 \mu \mathrm{m}$ polyimide as sensitive layer. The penetration depth inside the polyimide layer is $4.56 \mu \mathrm{m}$. The penetration depth value is not significantly influenced by the electrode finger width. However, it is correlated to the gap between the electrodes. In this case, the area above $4.6 \mu \mathrm{m}$ beyond the transducer surface does not contribute to the capacitance significantly. That is why the sensitivity of a $4.6 \mu \mathrm{m}$ thick polyimide layer and a $11 \mu \mathrm{m}$ layer are equal (as described in the previous Section 3.3). Generally, the sensing mechanism of the used material determines the ideal IDT structures. Smaller structures are better suited for thin layers. 

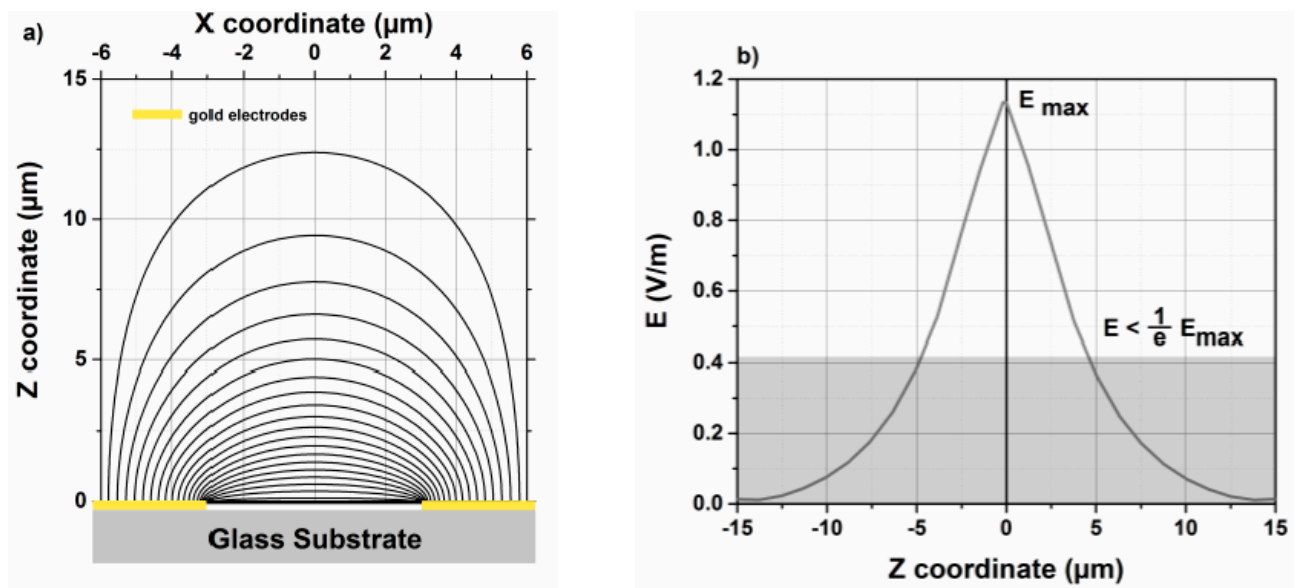

Figure 6. (a) Stream lines distribution between two adjacent electrodes on glass a substrate. The gold electrode width and the gap between two adjacent electrodes are fixed to $6 \mu \mathrm{m}$. (b) Electric field strength along a perpendicular axis to the IDT's planar surface and situated in the middle between two electrodes adjacent electrodes.

\subsection{Nanograss Polyimide Film}

In the last years, high aspect ratio structures from nature have inspired engineering and scientists to develop new materials. The nanostructures, having superhydrophobic surface properties, is still attracting considerable attention for application in photovoltaic [31], biosensors [32], and power source [33]. Simple methods to modify the polymeric layer structure have been developed, leading to superhydrophobic surfaces (water-repellent surfaces), without inducing a significant change in the chemistry of the polymeric layer.

In the case of polyimide material, Lee et al. proposed that etching of the layer at pressure equal to $100 \mathrm{mTorr}$ for $5 \mathrm{~min}$ results in a nanograss structure [20]. A pressure of $720 \mathrm{mTorr}$ was commonly used to obtain a more homogeneous surface feature [34]. This nanograss film showed a very small response time to moisture.

In the present work, the plasma etching process of polyimide films is carried out under oxygen plasma at two different pressures, 50 mTorr and 720 mTorr, using a reactive ion etching chamber from Plasmalab. Oxygen plasma is generated by a radio frequency (RF) power source. The RF power for both working pressures is fixed to $113 \mathrm{~W}$. The bias voltage, which built up between oxygen source and sample chuck, is $380 \mathrm{~V}$ and $140 \mathrm{~V}$ for lower and higher etching pressure, respectively. The bias voltage reflects the kinetic energy of the etching reactants. Hence, the oxygen ions with the lower pressure hit the polyimide surface with a higher kinetic energy. The etching rate of polyimide layer is determined by measuring the layer thickness by a profilometer after an etching time of 2 min. The plasma etched samples are analyzed by SEM, as presented in Figure 7. The thickness of both films is nearly equal. Both etching processes increase surface roughness and change the surface morphology of the polyimide layer. At the edge of the layer, both samples look like nanograss structures but the orientation of the fibers is different. In the inner area, both etched films consist of two layers. The bottom layer is a bulk polyimide layer that has not been affected by the etching process and the upper layer is a fiber-like layer. In case of the low pressure etched film, the nanograss covers $67 \%$ of the whole film thickness. In contrast, only $42 \%$ of the whole film thickness is structured in case of a higher-pressure plasma etching process.

Previous theoretical work established a correlation between pressure and etching rate [35] to explain the observation reported by SEM results. At high pressure, the number of collision events between oxygen ions inside the plasma increases drastically. Thus, the kinetic energy of the plasma ions decreases, which lowers the etching rate. At low pressures, the number of oxygen ions in the plasma is lower. Therefore, the number of reactants hitting the substrate surface decreases and the etch rate is 
decreased. As the number of collision between oxygen atoms during process is reduced, the reactants hit perpendicularly the polymer surface. For this reason, the fibers formed by the low pressure etching process are oriented perpendicularly to the substrate surface, more like grass. Contrary to the high pressure etching process, the fibers formed in the polymer layer are disordered, because the angles of incidence of the etching reactants are statistically distributed.
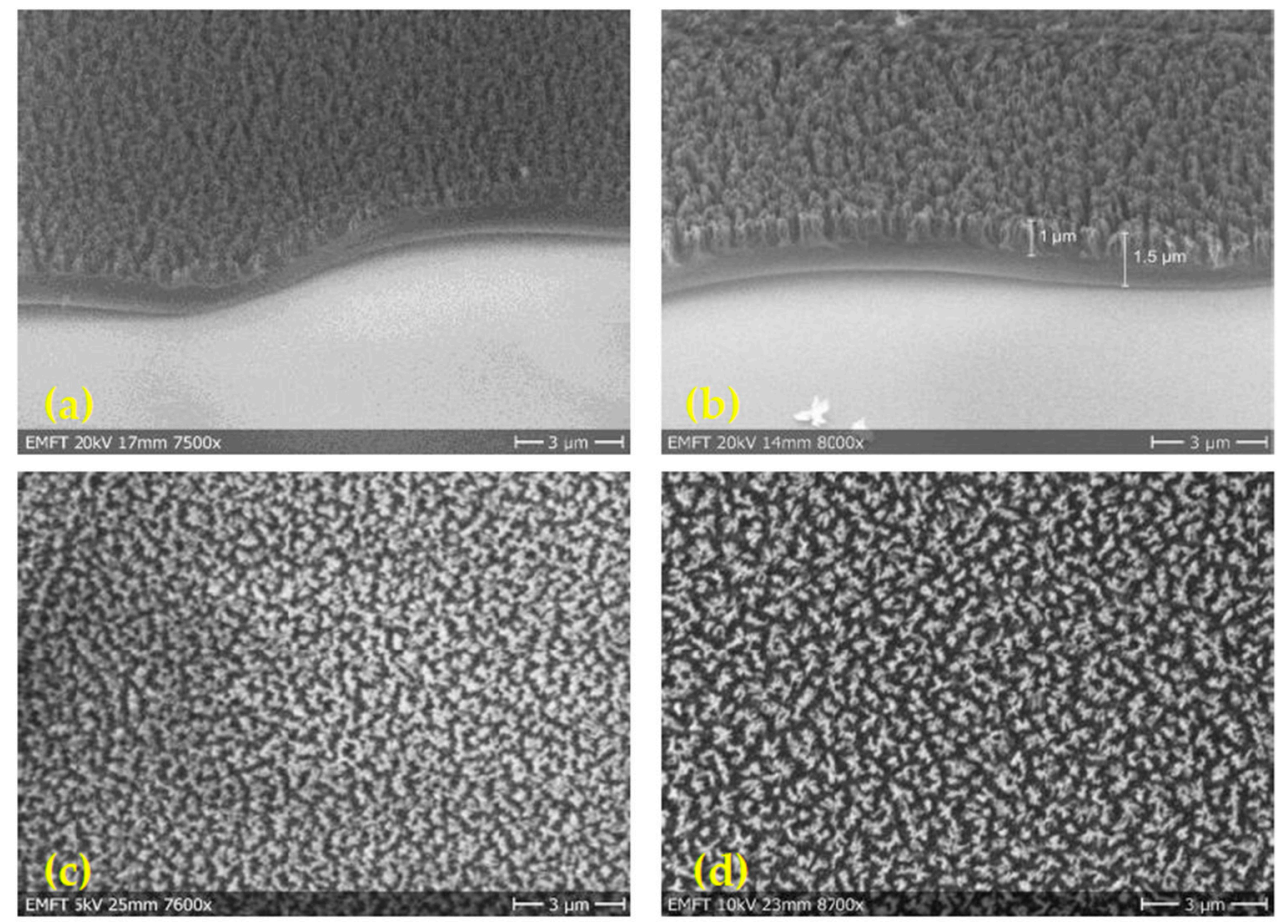

Figure 7. SEM cross-section pictures of plasma etched polyimide layers using different pressures: (a) $\mathrm{p}=720$ mTorr and (b) $\mathrm{p}=50$ mTorr. The thickness of the resulted grass structure is indicated by arrows. SEM top view pictures of etched polyimide layers at (c) $p=720 \mathrm{mTorr}$ resulting in a roughened surface with a surface coverage compared to (d) $p=50$ mTorr.

The realized etching process under oxygen plasma at low pressures, 50 mTorr, yields nanograss-like polyimide structures with higher etching rate [20]. In this study, the etching process results vertically oriented nanograss structures with $1 \mu \mathrm{m}$ height on the top of $0.5 \mu \mathrm{m}$ flat polyimide layer on the substrate. However, the dimensions of the individual fibers of the nanograss are comparable, around $50 \mathrm{~nm}$ in diameter with an interspacing distance of around $100 \mathrm{~nm}$.

\subsection{Humidity Sensor Performance}

A series of photosensitive polyimide layers are deposited onto IDTs. The layers present different thickness and surface morphologies. To characterize the performance of chemical sensors under development, sensors are placed into a hermitic test measurement chamber and the sensors capacitance is monitored under different humidity levels by an impedance analyzer. A commercial humidity sensor, SHT25 device with a standard calibration, is selected for reference.

\subsubsection{Dynamic Response Using Impedance Analyzer}

Figure 8a shows the measured capacitance with the impedance analyzer inside the sensor chamber, which is normalized versus time for polyimide material with different thickness and surface morphologies under different RH levels. The signal delivered by a commercial reference sensor, SHT25, operated under the same conditions, is superposed to the developed sensors data. The Figure $8 \mathrm{~b}$ shows the capacitance of the measured sensors in Figure $7 \mathrm{a}$, as a function of relative 
humidity measured by SHT25. The capacitance refers to the measured value after $30 \mathrm{~min}$ in a certain humidity. For all sensors, the calibration curves present a linear behaviour. In Table 1, sensitivity and linearity of developed sensors are summarized. The sensitivity is expresed as the ratio of capacitance to relative humidity:

$$
\text { Sensitivity }(f F / \% R H)=\frac{C_{R H=85 \%}-C_{R H=6 \%}}{R H_{85 \%}-R_{6 \%}}
$$

where: $C_{\mathrm{RH}=85 \%}$ and $\mathrm{C}_{\mathrm{RH}=6 \%}$ are the measured capacitance at high and low level of relative humidity, respectively.
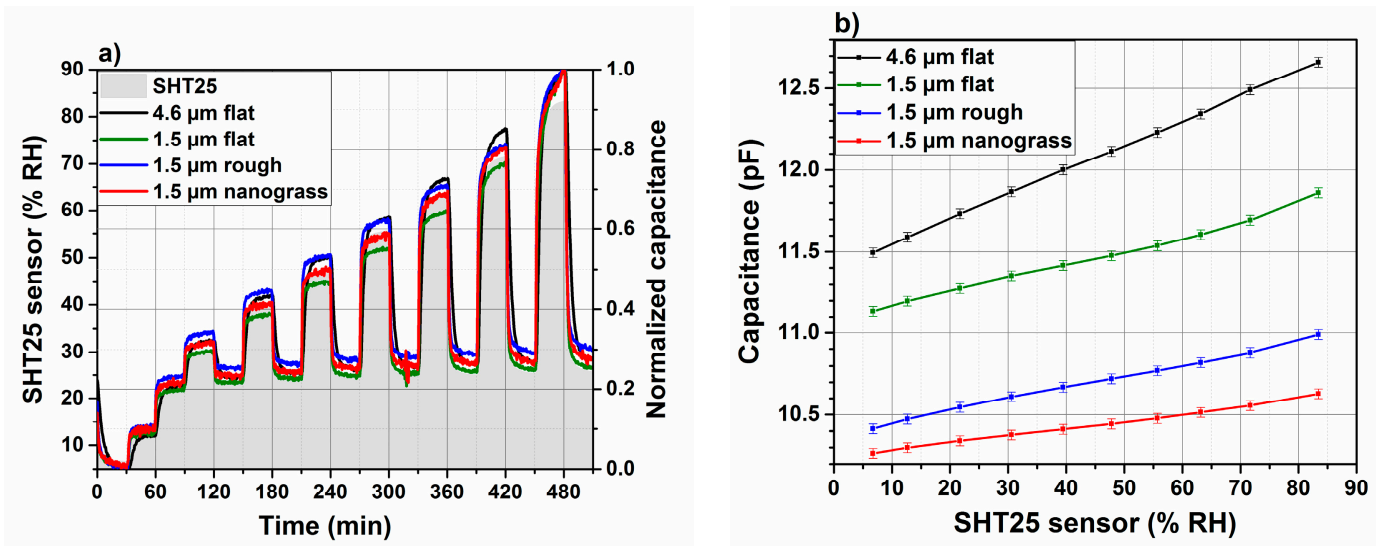

Figure 8. (a) Normalized capacitance of humidity sensors measured at $40 \mathrm{kHz}$ versus time under different RH levels and for different thicknesses and surface morphologies. (b) Calibration curves of developed humidity sensors with different thicknesses and surface morphologies as indicated.

Table 1. Sensitivity and linearity of the developed polyimide-based humidity sensor with different morphologies at $40 \mathrm{kHz}$ and $25^{\circ} \mathrm{C}$. The error of the impedance analyzer is $0.3 \mathrm{pF}$.

\begin{tabular}{ccccc}
\hline & $\mathbf{4 . 6} \boldsymbol{\mu m}$ Flat & $\mathbf{1 . 5} \boldsymbol{\mu m}$ Flat & $\mathbf{1 . 5} \boldsymbol{\mu m}$ Rough & $\mathbf{1 . 5} \boldsymbol{\mu m}$ Nanograss \\
\hline Sensitivity (fF/\% RH) & 15.2 & 9.48 & 7.47 & 4.75 \\
linearity & 0.999 & 0.995 & 0.998 & 0.998 \\
\hline
\end{tabular}

The sensitivity of the sensors decreases from 15 to $9.5 \mathrm{fF} / \% \mathrm{RH}$ by decreasing the polyimide thickness from 4.6 to $1.5 \mu \mathrm{m}$ with a flat morphology. As described in Section 3.4, the sensitive region of the transducer is situated at $4.6 \mu \mathrm{m}$ beyond the transducers surface. Obviously, by decreasing the polyimide layer thickness, the sensitivity also decreases. Increasing the roughness of a $1.5 \mu \mathrm{m}$ polyimide leads to a decrease in the sensor sensitivity. However, the calibration curve linearity is not affected. However, it was noted that a polyimide-based humidity sensor presents an increase in the sensitivity from 351 to $506 \mathrm{fF} / \% \mathrm{RH}$ if the the layer thickness is etched from 400 to $100 \mathrm{~nm}$ under $\mathrm{O} 2$ plasma [27]. This improvement in the sensitivity is related to the designed electrodes, which are not planar but parallel. The bottom electrode is deposited in a substrate cavity and the top electrode has a comb shape. The polyimide layer is sandwiched between the bottom and the top electrodes. Such parallel electrodes configuration is often related to high sensitivity [26,27].

\subsubsection{Response and Recovery Times Using Evaluation Kit}

Although a gas measurement setup, combining the gas mixing apparatus and electrical measurements using impedance analyzer, is a standard method to characterize the performance of chemical sensors under development, the response and recovery times, typically less than 3 min, are difficult to be accurately monitored for RH sensors under test. The reason is that some minutes ( $5 \mathrm{~min}$ ) are needed to ensure a complete gas exchange inside the testing chamber [28]. To overcome the issue of studying the response and recovery times of the developed humidity sensors, a miniaturized 
evaluation kit is developed as a complementary setup. This evaluation kit is considered as a complementary tool to monitor simultaneously the electrical characteristic of the sensors and to measure their response and recovery times. Moreover, it can also be used to examine the sensor's performance in more realistic conditions and to study its long-term stability. The gas mixing apparatus is used to provide a humid test gas. A second gas flow of dry carrier gas was provided by a separate mass flow controller. A Luer Lock vent is used to switch between the humid and the dry test gas. Hence, the gas exchange time and the measurement interval are fixed to $400 \mathrm{~ms}$ and $150 \mathrm{~ms}$, respectively.

The response and recovery times in this study are defined as the times required for the sensor capacitance to reach $90 \%$ of the maximum measured signal, when the humidity is changed between two humidity levels (here from 6\% RH to $67 \%$ ). Figure 9 summarizes the response and recovery times of three different developed humidity sensors having different polyimide film thicknesses $(11 \mu \mathrm{m}$ and $1.5 \mu \mathrm{m}$ ) and morphologies (flat, rough, and nanograss). It is worth noting that $1.5 \mu \mathrm{m}$ flat polyimide sensors have been excluded from further investigations, because their response and recovery times are higher as compared to the rough film with the same thickness.
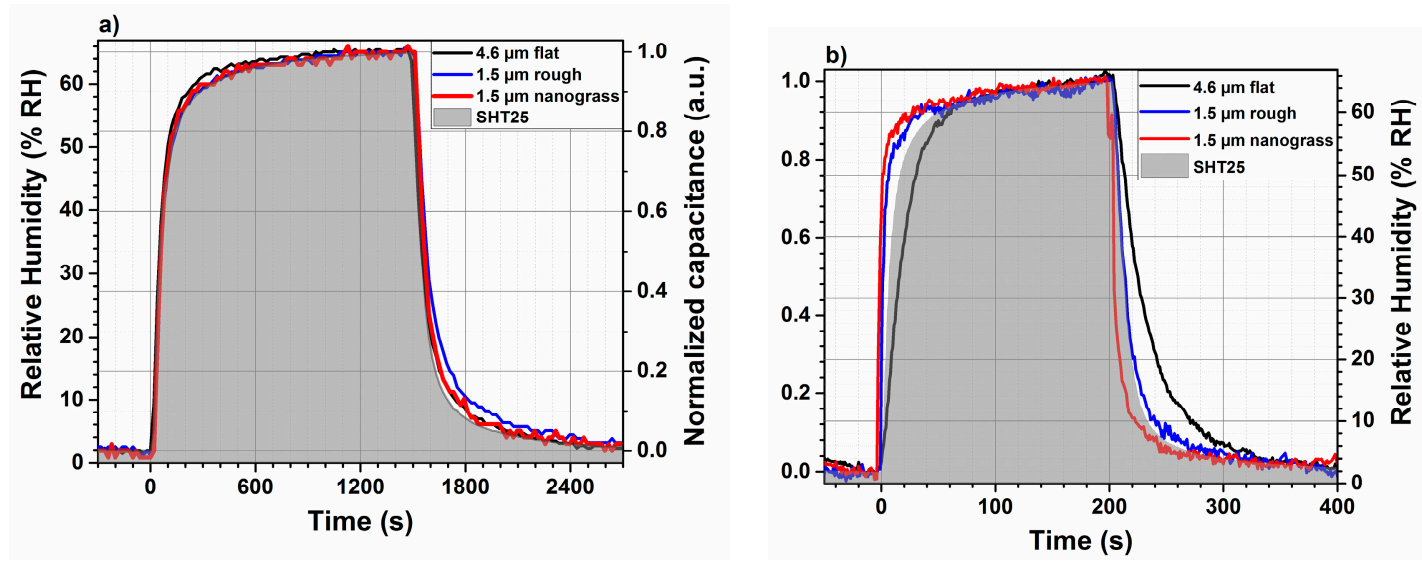

Figure 9. Response and recovery times of relative humidity sensor compared to a commercial one (SHT25) measured inside the chamber of the (a) gas mixing setup at $40 \mathrm{kHz}$ (b) evaluation kit.

As the sensing mechanism is based on diffusion, the thinner sensing layer shows a smaller response as well as a smaller recovery time. However, the nanograss-like layer shows the best performance even in comparison to commercial humidity sensors. An optimization of the transducer design and its miniaturization will lead to a further improved sensor performance. The measured capacitance, normalized, of the polyimide-based RH sensor (for both thicknesses and morphologies) as a function of time is shown in Figure 9. The relative humidity is determined by a commercial humidity sensor SHT25, placed in the same humidity condition as the developed ones.

The RH level in the test chamber is varied from dry to $65 \%$ and back to the initial level $6 \%$ after some seconds. The sensor samples with a polyimide thickness of $4.6 \mu \mathrm{m}$ having a flat morphology and $1.5 \mu \mathrm{m}$ rough surface and $1.5 \mu \mathrm{m}$ nanograss-like surface present the same response and recovery times once measured in the chamber of gas mixing setup. As mentioned in Section 2, the evaluation kit is more appropriate to deduce response and recovery times of sensors. Figure $9 \mathrm{~b}$ reports the response time and recovery times of the prepared samples with different thickness and morphologies. The sensors are placed into the chamber of the evaluation kit, which is connected to the gas mixing unit. The $1.5 \mu \mathrm{m}$ nanograss polyimide shows the lowest response time of about $18 \mathrm{~s}$ in comparison to the same thickness of rough polyimide layer $(1.5 \mu \mathrm{m}$ rough) and $4.6 \mu \mathrm{m}$ flat layer and the commercial sensor; see Table 2. Regarding the response time, the same observation is valid for nanograss film. This means that the recovery time of a nanograss film is $31 \mathrm{~s}$, which is lower than $1.5 \mu \mathrm{m}$ rough polyimide layer (45 s), $4.6 \mu \mathrm{m}$ flat layer ( $80 \mathrm{~s})$, and the commercial sensor (40 s). The extremely rapid response and recovery times for the sensing layer having a rough and nanograss morphology is 
probably related to the porous structure in comparison to the flat morphology. Such porous structure of the sensing layer, created during the plasma etch process, permits a rapid diffusion of water molecule inside and outside the matrix. Besides this, the layer thickness is another important parameter, which should be optimized for the chosen interdigitated electrodes. The same observation regarding the response and recovery times were noted for different polyimide (Kapton HN50) thicknesses [25]. The response time was decreases from $25 \mathrm{~min}$ to $4.75 \mathrm{~min}$ by decreasing the Kapton HN50 thickness respectively from $25 \mu \mathrm{m}$ to $7.5 \mu \mathrm{m}$. In our case of polyimide-based humidity sensors, an increase in the recovery time in comparison to a response time of $50 \%$ to $70 \%$ was obtained, which is higher than the calculated value $26 \%$ from other work [25]. However, the commercial sensor shows the same response and recovery times.

Table 2. Response and recovery times of polyimide-based humidity sensor with different morphologies. The error bar is $2 \%$ due to reference capacitor and analog-digital converter (ADC).

\begin{tabular}{ccccc}
\hline & RH Sensor (SHT 25) & $\mathbf{4 . 6} \boldsymbol{\mu m}$ Flat & $\mathbf{1 . 5} \boldsymbol{\mu m}$ Rough & $\mathbf{1 . 5} \boldsymbol{\mu m}$ Nanograss \\
\hline Response time $\tau 90_{\text {res }}(\mathrm{s})$ & 42 & 54 & 26 & 18 \\
Recovery time $\tau 90_{\text {rec }}(\mathrm{s})$ & 40 & 80 & 45 & 31 \\
Increase in $\tau 90_{\text {rec }}$ Compared to & -5 & 48 & 73 & 72 \\
$\tau 90_{\text {res }}\left(\frac{\tau_{\text {rec }}}{\tau_{\text {res }}}-1\right) * 100 \%$ & & & & \\
\hline
\end{tabular}

It is worth noting that the sensor responses are uncorrelated to the used ambient air. No significant differences are detected of measuring the sensors response under carrier gases $\mathrm{N}_{2}$ or synthetic air.

Generally, the adsorption and desorption of a specific analyte carried by an inert gas into a sensing material matrix is governed by either diffusion process or thermodynamically controlled process or even both processes. In case of an ideal diffusion-controlled process, the response time and the recovery time should be equal. In the thermodynamically controlled process, the adsorption is an exothermic process, whereas desorption is an endothermic process. Consequently, desorption requires higher external energy which is reflected by a higher recovery time. An adsorbed molecule on the surface of a sensing layer at certain temperature remains stable at this state. A moderate increase of the sensor temperature, in the absence of any interaction between the sensing material and the analyte, leads to desorption of the desorbed molecule from the surface and returns into its initial gas form.

As confirmed from the FTIR measurements, the polyimide material presents no chemical interaction between the polymer backbone and water molecules. However, the polyimide layer with rough and nanograss-like morphologies show a large difference between their response and recovery times $(72 \%)$ to humidity. This large difference between the two parameters describing the humidity sensor is probably due to structure changes into the polyimide matrix induced by the plasma process. These structure changes in the polymer induced by the $\mathrm{O}_{2}$ plasma etch will be subject of future studies.

\section{Conclusions}

In summary, a systematic examination of the sensor performance of a series of polyimide layers with different thickness and morphologies (flat, etched, and nanograss) exposed to different humidity levels are measured simultaneously in the same test chambers. We have successfully demonstrated that the nanostructure of polyimide to obtain nanograss can be well controlled by adjustment of a series of $\mathrm{O}_{2}$ plasma etch parameters. The nanograss morphology exhibits the best performance among the studied morphology types. The ideal polyimide layer thickness turns out to be equal to the penetration depth of the used interdigitated transducer. The sensor signal, capacitance, follows a linear dependence with RH. Polyimide sensing layers with nanograss morphology ensure the best humidity sensor performance regarding the response and recovery times. The optimal polyimide layer thickness 
and morphology can be chosen as a function of the envisaged application of relative humidity sensors and the used interdigitated transducer.

Author Contributions: J.B. designed the experiments based on previous realized work and headed the research project. M.S. did the experimental work and FEM simulation. A.D. did the technological realization of IDTs and polyimide coating and etching. H.-E.E. and M.S. realized the electronic system "evaluation board". P.M.-B. provided FTIR characterization and participated actively to discussing the results. H-E.E. and C.K. participated actively to constructive discussions and to the method of measurements. J.B. and M.S. co-wrote the paper with input from all authors. All authors discussed and commented on the manuscript.

Acknowledgments: The authors wish to thank Vu Chi. Hung, R. Faul, and D. Linke for designing, technological fabricating, cleaning, and wire bonding of IDTs. The authors wish to thank G.A.M. Nastasi for providing basic concepts of the microcontroller setup. This work was partly financially supported by the Bavarian Ministry of Economic Affairs and Media, Energy and Technology, Germany, within the project "Sensorplattform für-Condition Monitoring".

Conflicts of Interest: The authors declare no conflict of interest.

\section{References}

1. Fraden, J. Handbook of Modern Sensors: Physics, Designs, and Applications, 3rd ed.; Springer: New York, NY, USA, 2004.

2. Faia, P.M.; Jesus, E.L.; Louro, C.S. $\mathrm{TiO}_{2}$ : $\mathrm{WO}_{3}$ composite humidity sensors doped with $\mathrm{ZnO}$ and $\mathrm{CuO}$ investigated by impedance spectroscopy. Sens. Actuators B Chem. 2014, 203, 340-348. [CrossRef]

3. Tripathy, A.; Pramanik, S.; Manna, A.; Shasmin, H.N.; Radzi, Z.; Abu Osman, N.A. Uniformly Porous Nanocrystalline CaMgFe $1.33 \mathrm{Ti}_{3} \mathrm{O}_{12}$ Ceramic Derived Electro-Ceramic Nanocomposite for Impedance Type Humidity Sensor. Sensors 2016, 16, 2029.

4. Tripathy, A.; Pramanik, S.; Manna, A.; Bhuyan, S.; Azrin Shah, N.F.; Radzi, Z.; Abu Osman, N.A. Design and Development for Capacitive Humidity Sensor Applications of Lead-Free Ca,Mg,Fe,Ti-Oxides-Based Electro-Ceramics with Improved Sensing Properties via Physisorption. Sensors 2016, 16, 1135. [CrossRef] [PubMed]

5. Dinç Zor, Ş.; Cankurtaran, H. Impedimetric Humidity Sensor Based on Nanohybrid Composite of Conducting Poly(diphenylamine sulfonic acid). J. Sens. 2016, 2016, 5479092. [CrossRef]

6. Ni, K.; Chan, C.C.; Chen, L.; Dong, X.; Huang, R.; Ma, Q. A chitosan-coated humidity sensor based on Mach-Zehnder interferometer with waist-enlarged fusion bitapers. Opt. Fiber Technol. 2017, 33, 56-59. [CrossRef]

7. Chen, Z.; Lu, C. Humidity Sensors: A Review of Materials and Mechanisms. Sens. Lett. 2005, 3, $274-295$. [CrossRef]

8. Çiğil, A.B.; Cankurtaran, H.; Kahraman, M.V. Photo-crosslinked thiol-ene based hybrid polymeric sensor for humidity detection. React. Funct. Polym. 2017, 114, 75-85. [CrossRef]

9. Adhikari, B.; Majumdar, S. Polymers in sensor applications. Prog. Polym. Sci. 2004, 29, 699-766. [CrossRef]

10. Bai, H.; Shi, G. Gas Sensors Based on Conducting Polymers. Sensors 2007, 7, 267-307. [CrossRef]

11. McCafferty, E.; Zettlemoyer, A.C. Adsorption of water vapour on alpha- $\mathrm{Fe}_{2} \mathrm{O}_{3}$. Discuss. Faraday Soc. 1971, 52, 239-254. [CrossRef]

12. Morimoto, T.; Nagao, M.; Tokuda, F. Relation between the amounts of chemisorbed and physisorbed water on metal oxides. J. Phys. Chem. 1969, 73, 243-248. [CrossRef]

13. De Grotthuss, C.J.T. Memoir on the decomposition of water and of the bodies that it holds in solution by means of galvanic electricity. Biochim. Biophys. Acta 2006, 1757, 871-875. [CrossRef] [PubMed]

14. Farahani, H.; Wagiran, R.; Hamidon, M.N. Humidity sensors principle, mechanism, and fabrication technologies: A comprehensive review. Sensors 2014, 14, 7881-7939. [CrossRef] [PubMed]

15. Yamazoe, N.; Sakai, G.; Shimanoe, K. Oxide Semiconductor Gas Sensors. Catal. Surv. Asia 2003, 7, 63-75. [CrossRef]

16. Li, G.Q.; Laib, P.T.; Zeng, S.H.; Huang, M.Q.; Cheng, Y.C. Photo-, thermal and humidity sensitivity characteristics of $\mathrm{Sr}_{1-x} \mathrm{La}^{x} \mathrm{TiO}_{3}$ film on $\mathrm{SiO}_{2} / \mathrm{Si}$ substrate. Sens. Actuators A Phys. 1997, 63, 223-226. [CrossRef]

17. Yamazoe, N.; Fuchigami, J.; Kishikawa, M.; Seiyama, T. Interactions of tin oxide surface with $\mathrm{O}_{2}, \mathrm{H}_{2} \mathrm{O}$ and $\mathrm{H}_{2}$. Surf. Sci. 1979, 86, 335-344. [CrossRef] 
18. Sakai, Y.; Sadaoka, Y.; Matsuguchi, M. Humidity sensors based on polymer thin films. Sens. Actuators B Chem. 1996, 35, 85-90. [CrossRef]

19. Sakai, Y.; Matsuguchi, M.; Hurukawa, T. Humidity sensor using cross-linked poly(chloromethyl styrene). Sens. Actuators B Chem. 2000, 66, 135-138. [CrossRef]

20. Lee, H.; Lee, S.; Jung, S.; Lee, J. Nano-grass polyimide-based humidity sensors. Sens. Actuators B Chem. 2011, 154, 2-8. [CrossRef]

21. Gu, L.; Huang, Q.-A.; Qin, M. A novel capacitive-type humidity sensor using CMOS fabrication technology. Sens. Actuators B Chem. 2004, 99, 491-498. [CrossRef]

22. Laconte, J.; Wilmart, V.; Flandre, D.; Raskin, J.-P. High-sensitivity capacitive humidity sensor using 3-layer patterned polyimide sensing film. In Proceedings of the IEEE Sensors 2003, Toronto, ON, Canada, 22-24 October 2003; pp. 372-377, IEEE Cat. No.03CH37498.

23. Virtanen, J.; Ukkonen, L.; Björninen, T.; Sydänheimo, L. Printed humidity sensor for UHF RFID systems. In Proceedings of the IEEE Sensors Applications Symposium (SAS), Limerick, Ireland, 23-25 February 2010; pp. 269-272.

24. Rivadeneyra, A.; Fernández-Salmerón, J.; Agudo-Acemel, M.; López-Villanueva, J.A.; Capitan-Vallvey, L.F.; Palma, A.J. Printed electrodes structures as capacitive humidity sensors: A comparison. Sens. Actuators A 2016, 244, 56-65. [CrossRef]

25. Harrey, P.M.; Ramsey, B.J.; Evans, P.S.A.; Harrison, D.J. Capacitive-type humidity sensors fabricated using the offset lithographic printing process. Sens. Actuators B 2002, 87, 226-232. [CrossRef]

26. Choi, K.S.; Kim, D.S.; Yang, H.J.; Ryu, M.S.; Chang, S.P. A highly sensitive humidity sensor with a novel hole array structure using a polyimide sensing layer. RSC Adv. 2014, 4, 32075. [CrossRef]

27. Kim, J.-H.; Hong, S.-M.; Moon, B.-M.; Kim, K. High-performance capacitive humidity sensor with novel electrode and polyimide layer based on MEMS technology. Microsyst. Technol. 2010, 16, 2017-2021. [CrossRef]

28. Endres, H.-E.; Jander, H.D.; Göttler, W. A test system for gas sensors. Sens. Actuators B Chem. 1995, 23, 163-172. [CrossRef]

29. Endres, H.-E.; Drost, S.; Hutter, F. Impedance spectroscopy on dielectric gas sensors. Sens. Actuators B Chem. 1994, 22, 7-11. [CrossRef]

30. Nastasi, G.; Scuderi, A.; Endres, H.-E.; Hell, W.; Bock, K. Simple Cost Effective and Network Compatible Readout for Capacitive and Resistive (Chemical) Sensors. Procedia Eng. 2014, 87, 1234-1238. [CrossRef]

31. Agarwal, N.; Ponoth, S.; Plawsky, J.; Persans, P.D. Roughness evolution in polyimide films during plasma etching. Appl. Phys. Lett. 2001, 78, 2294-2296. [CrossRef]

32. Verdonck, P. Plasma Etching. Oficina de Microfabrição: Projeto e Construção de CI's MOS; UNICAMP, Campinas: Sao Paulo, Brazil, 2006; Chapter 10.

33. Zhang, Y.; Diao, Y.; Lee, H.; Mirabito, T.J.; Johnson, R.W.; Puodziukynaite, E.; John, J.; Carter, K.R.; Emrick, T.; Mannsfeld, S.C.B.; et al. Intrinsic and extrinsic parameters for controlling the growth of organic single-crystalline nanopillars in photovoltaics. Nano Lett. 2014, 14, 5547-5554. [CrossRef] [PubMed]

34. Hong, C.-C.; Huang, S.-Y.; Shieh, J.; Chen, S.-H. Enhanced Piezoelectricity of Nanoimprinted Sub-20 nm Poly(vinylidene fluoride-trifluoroethylene) Copolymer Nanograss. Macromolecules 2012, 45, 1580-1586. [CrossRef]

35. Romanowicz, B.F.; Laudon, M. 2005 NSTI Nanotechnology Conference and Trade Show: NSTI Nanotech 2005, Anaheim, CA, USA, 8-12 May 2005; NSTI: Cambridge, MA, USA, 2005.

(C) 2018 by the authors. Licensee MDPI, Basel, Switzerland. This article is an open access article distributed under the terms and conditions of the Creative Commons Attribution (CC BY) license (http://creativecommons.org/licenses/by/4.0/). 